\title{
Verzögerung von Komplikationen unter Denosumab bestätigt
}

\begin{abstract}
Die Ergebnisse einer integrierten Analyse von 3 Phase-III-Studien zum Vergleich von Denosumab und Zoledronsäure bei Krebspatienten mit Knochenmetastasen bestätigen die in den einzelnen Studien gezeigten Vorteile des Antikörpers (Lipton A et al., 2010, ESMO:\#1249, poster presentation).
\end{abstract}

Sie basieren auf der Untersuchung von Knochenkomplikationen bei mehr als $5.700 \mathrm{~Pa}$ tienten mit Mamma- oder Prostatakarzinom bzw. anderen soliden Tumoren oder multiplem Myelom und mindestens einer Knochenmetastase. Die Patienten hatten in den 3 Phase-III-Studien jeweils alle 4 Wochen $120 \mathrm{mg}$ Denosumab s.c. oder $4 \mathrm{mg}$ Zoledronsäure i.v. für 30 Monate erhalten.

\section{Deutlich verbesserte Lebensqualität}

Bei der Dauer bis zum Auftreten der ersten Knochenkomplikation sowie der Dauer bis zum Auftreten der ersten und weiterer Knochenkomplikationen zeigte sich die Überlegenheit von Denosumab im Vergleich zu Zoledronsäure: Die mediane Zeit bis zum Auftreten der ersten Knochenkomplikation betrug unter Denosumab 27,7 Monate, unter Zoledronsäure 19,4 Monate (Hazard-Ratio [HR] 0,83; $<<0,001)$. Bei der Dauer bis zum Auftreten der ersten und weiterer Knochenkomplikationen ergab sich beim Vergleich von Denosumab versus Zoledronsäure eine Risikoreduktion um 18\% (HR 0,82; $\mathrm{p}<0,001)$.

Wie Allan Lipton, Pennsylvania/USA, betonte, konnte zwar kein Überlebens- oder Progressionsvorteil festgestellt werden. Die Patienten benötigten jedoch unter der Denosumab-Therapie weniger Analgetika als die Patienten in der Vergleichsgruppe. Die Lebensqualität hatte sich deutlich verbessert. UF

Quelle: Veranstaltung der Amgen $\mathrm{GmbH}$

\section{Geriatrische Onkologie}

\section{Versorgungssituation älterer Patienten}

\begin{abstract}
Aufgrund des demographischen Wandels werden Tumorerkrankungen in Zukunft zunehmen. Die Versorgungssituation der Patienten wird sowohl in medizinischer als auch in gesellschaftspolitischer, ethischer und ökonomischer Hinsicht eine Herausforderung darstellen.
\end{abstract}

Wie Ursula Lehr, Bonn-Bad Godesberg, betonte, muss eine optimale Behandlung auch bei älteren Patienten mit einer Tumorerkrankung gewährleistet sein. Die Tumortherapie sollte an den individuellen Gegebenheiten orientiert sein und die verbleibende Lebenserwartung des Patienten berücksichtigen.

Individuell angepasste Tumortherapie Für Patienten, die älter als 65 Jahre sind, gibt es derzeit jedoch keine evidenzbasier- ten Therapieempfehlungen. Friedrich Overkamp, Recklinghausen, zufolge weisen die meisten Zytostatika und insbesondere die modernen, innovativen Tumortherapeutika aus der Gruppe der zielgerichteten Therapien, wiez.B. Antikörper und Tyrosinkinaseinhibitoren, bei älteren Patienten nach bislang vorliegenden Daten keine erhöhte Toxizität auf. Demzufolge können ältere Patienten von diesen Medikamenten ebenso profitieren wie jüngere Patienten. Nicht das kalendarische Alter, sondern der Funktionsstatus der Organe des Patienten - insbesondere der Leber und der Nieren - sei dafür verantwortlich, wie gut eine Therapie vertragen wird und inwieweit die therapeutischen Erwartungen zu realisieren sind, so Overkamp. HAF

Quelle: Veranstaltung der Roche Pharma AG

\section{Adressverzeichnis} Psychoonkologie

Die Initiative „Psyche hilft Körper" wurde von der GlaxoSmithKline GmbH \& Co. KG ins Leben gerufen, um über die Bedeutung der psychoonkologischen Therapie von Krebserkrankungen zu informieren. Als eine der ersten Aktionen ist das bundesweite Adressverzeichnis von Psychoonkologen "Sprechstunde für die Seele" erhältlich. Die Fachärzte sind nach Postleitzahlen geordnet. Patienten sehen an der farblichen Kennzeichnung, welche Therapie der Arzt anbietet oder welche Weiterbildung er hat. Auch die Adressen von Selbsthilfeorganisationen und Fachverbänden sind enthalten. Das Adressverzeichnis kann unter www.gsk-onkologie. de in der Rubrik "Informationsangebote für Patienten“ heruntergeladen werden.

\section{Filgrastim-Biosimilar}

Mit dem Filgrastim-Biosimilar Nivestim ${ }^{\mathrm{TM}}$ wurde das zweite Biosimilar der Hospira Deutschland $\mathrm{GmbH}$ von der Europäischen Kommission zugelassen. Das Entwicklungsprogramm für dieses Biosimilar wurde gemäß den Richtlinien der Europäischen Arzneimittelagentur durchgeführt, um die Vergleichbarkeit mit dem Referenzprodukt in Bezug auf die Verträglichkeit, Wirksamkeit und Qualität zu gewährleisten.

\section{Sekundäre Pflanzenstoffe und Mikronährstoffe}

Sekundäre Pflanzenstoffe können eine nützliche Ergänzung bei der Therapie von Tumorpatienten sein. So weisen Studienergebnisse darauf hin, dass sekundäre Pflanzenstoffe auf Tumorzellen eine hemmende Wirkung ausüben können (Sarkar FH et al., 2009, Cancer Treat Rev 35: 597-607). Besonders die Kombination bestimmter sekundärer Pflanzenstoffe kann das Wachstum von Tumorzellen reduzieren (Somers-Edgar TJ et al., 2008, Int J Cancer 122: 1966-1971). Zusätzlich ist nach Informationen der Orthomol GmbH die Supplementation von Mikronährstoffen wichtig, um Mikronährstoffdefizite bei Tumorpatienten auszugleichen. 